Pacific Journal of Mathematics

QUASI-POSITIVE OPERATORS 


\section{QUASI-POSITIVE OPERATORS}

\section{W. SASSER}

1. Introduction. The classical results of Perron and Frobenius ([6], [7], [12]) assert that a finite dimensional, nonnegative, non-nilpotent matrix has a positive eigenvalue which is not exceeded in absolute value by any other eigenvalue and the matrix has a nonnegative eigenvector corresponding to this positive eigenvalue. If the matrix has strictly positive entries, then there is a positive eigenvalue which exceeds every other eigenvalue in absolute value, and the corresponding space of eigenvectors is one-dimensional and is spanned by a vector with strictly positive coordinates. Numerous generalizations of these results to order-preserving linear operators acting in ordered linear spaces have appeared in recent years; a short bibliography is included at the end of this paper. In this paper a generalization in a different direction is obtained which reduces, in the finite dimensional case, to the assertion that the Perron-Frobenius theorems hold if it is only required that all but a finite number of the powers of the matrix satisfy the given conditions. The principal results are theorems of the Perron-Frobenius type which are applicable to any compact linear operator (the compactness condition is weakened somewhat), acting in an ordered real Banach space $B$, which satisfies a condition weaker than order-preserving. In addition, the results apply to the case when the "cone" of positive elements in $B$ has no interior.

2. Preliminaries. Throughout the sequel, $B$ will denote a real Banach space with norm $\|\cdot\|$. The complex extension of $B, \widetilde{B}$, is the complex Banach space $\widetilde{B}=\{x+i y \mid x, y \in B\}$ with the obvious definitions of addition and complex scalar multiplication and the norm in $\widetilde{B}$ is $\|x+i y\|=\sup _{\theta}\|\cos \theta \cdot x+\sin \theta \cdot y\|$. If $T$ is a (real) linear operator on $B$ into $B$, the (complex) linear operator $\widetilde{T}$ on $\widetilde{B}$ into $\widetilde{B}$ is defined by $\widetilde{T}(x+i y)=T x+i T y . \quad T$ is bounded if and only if $\widetilde{T}$ is bounded, in which case $\|T\|=\|\widetilde{T}\|$. The spectrum, $\sigma(T)$, and the resolvent, $\rho(T)$, are defined to be the corresponding sets associated with the operator $\widetilde{T}$. We denote the spectral radius of $T$ by $r_{T}, r_{T}=$ $\lim _{n \rightarrow \infty}\left\|T^{n}\right\|^{1 / n}=\sup _{\lambda \in \sigma(T)}|\lambda|$ (provided $\|T\|<\infty$ ).

In all of our results there will be a basic assumption that the linear operator under consideration is quasi-compact, a notion which we will now define. A bounded linear operator $T$ is compact (also called completely continuous) if each sequence $T x_{1}, T x_{2}, \cdots$, with

Received September 13, 1962. This work was performed under the auspices of the Inited States Atomic Energy Commission. 
$\left\|x_{i}\right\| \leqq 1, i=1,2, \cdots$, has a convergent subsequence. $T$ is quasicompact if there exists a positive integer $n$ and a bounded linear operator $V$ such that $T^{n}-V$ is compact and $r_{V}<r_{T}^{n}{ }^{1}{ }^{1}$ There are a number of properties possessed by quasi-compact operators some of which we state now without proof. ${ }^{2}$ If $\lambda_{0} \in \sigma(T)$ and $\left|\lambda_{0}\right|=r_{T}$, then $\lambda_{0}$ is an isolated point in $\sigma(T)$ and is in the point spectrum, i.e., $\left(\lambda_{0} I-\widetilde{T}\right)$ is not one-to-one. The resolvent operator, $R(\lambda, T) \equiv(\lambda I-\widetilde{T})^{-1}$, exists in a neighborhood of $\lambda_{0}$ (excluding $\lambda_{0}$ ) and, in this neighborhood, $R(\lambda, T)$ has a Laurent series expansion of the form

$$
R(\lambda, T)=\sum_{k=1}^{n\left(\lambda_{0}\right)} \frac{\left(\lambda_{0} I-\widetilde{T}\right)^{k-1}}{\left(\lambda-\lambda_{0}\right)^{k}} P\left(\lambda_{0}, T\right)+\sum_{k=0}^{\infty}\left(\lambda-\lambda_{0}\right)^{k} A_{k}\left(\lambda_{0}, T\right)
$$

where $A_{k}\left(\lambda_{0}, T\right)$ is a bounded linear operator and the series on the right is convergent in the uniform operator topology. The integer $n\left(\lambda_{0}\right)$ is the index of $\lambda_{0}$, i.e., $n\left(\lambda_{0}\right)$ is the smallest integer $n$ such that $\left\{x \mid\left(\lambda_{0} I-\widetilde{T}\right)^{n+1} x=0\right\}=\left\{x \mid\left(\lambda_{0} I-\widetilde{T}\right)^{n} x=0\right\} . \quad P\left(\lambda_{0}, T\right)$ is a projection onto the finite dimensional space $\left\{x \mid\left(\lambda_{0} I-\widetilde{T}\right)^{n\left(\lambda_{0}\right)} x=0\right\}$. The minimal property of $n\left(\lambda_{0}\right)$ implies that $\left(\lambda_{0} I-\widetilde{T}\right)^{n\left(\lambda_{0}\right)-1} P\left(\lambda_{0}, T\right) \neq 0$.

We recall that for an arbitrary bounded linear operator, the resolvent $R(\lambda, T)=(\lambda I-\widetilde{T})^{-1}$ is an analytic function of $\lambda$ for $\lambda \in \rho(T)$ and the expansion $R(\lambda, T)=\sum_{k=0}^{\infty}(1 / \lambda)^{k+1} \widetilde{T}^{k}$ is valid for $|\lambda|>r_{T}$.

3. Quasi-positive operators. A cone in $B$ is a convex set $K$ which contains $\lambda x$ for all $\lambda \geqq 0$ if it contains $x$. $K$ is a proper cone if $x \in K$ and $-x \in K$ imply $x=0$. A cone $K$ induces an ordering $\geqq$ in $B$ with $x \geqq y$ if and only if $x-y \in K$. This transitive ordering. satisfies

(1) if $x \geqq y, u \geqq v$, then $x+u \geqq y+v$,

(2) if $x \geqq y$ and $\lambda \geqq 0$, then $\lambda x \geqq \lambda y$, and

(3) $x \geqq y$ if and only if $-y \geqq-x$.

If the cone is proper, then the ordering satisfies, in addition,

(4) if $x \geqq y$ and $y \geqq x$, then $x=y$.

We will use the notation $x>y$ to denote $x \geqq y, x \neq y$. Associated with a cone $K$ is a closed cone $K^{+}$in the conjugate space $B^{*}$ of continuous, real-valued, linear functions on $B$, consisting of those $x^{*} \in B^{*}$ with the property that $x^{*}(x) \geqq 0$ for all $x \in K . K^{+}$is a proper cone if and only if the linear space spanned by $K$ is dense in $B$ (a set with this property is called fundamental). This is an easy consequence of the Hahn-Banach theorem on the extension of linear functionals. We will use the notations $x^{*} \geqq y^{*}$ and $x^{*}>y^{*}$ to denote $x^{*}-y^{*} \in K^{+}$

1 Note that a compact operator is quasi-compact if and only if it has a positive spectral radius.

${ }^{2}$ For details, see Yu. L. Smvl'yan, Completely continuous perturbations of operators, Amer. Math. Soc. Translations 10, 341-344. 
and $x^{*}-y^{*} \in K^{+}, x^{*} \neq y^{*}$, respectively. An element $x>0 \quad\left(x^{*}>0\right)$ will be called strictly positive if $x^{*}(x)>0$ for all $x^{*}>0\left(x^{*}(x)>0\right.$ for all $x>0$ ).

The following theorem is a characterization of a closed cone and its interior (when the latter is nonvoid) in terms of $K^{+}$. The proof may be found, for example, in [11] (Theorem 1.3 and its corollaries, pg. 16).

THEOREM 1. Let $K$ be a closed cone in $B$. Then $x \in K$ if and only if $x^{*}(x) \geqq 0$ for all $x^{*} \geqq 0$. If $K$ has a nonvoid interior, then

(1) $x$ is in the interior of $K$ if and only if $x$ is strictly positive and

(2) for each $x$ on the boundary of $K$ there exists an $x^{*}>0$ such that $x^{*}(x)=0$.

CoRollary. If $K$ is a closed proper cone, $K^{+}$is a total set of functionals, i.e., for each $x \neq 0, x \in B$, there exists $x^{*}>0$ such that $x^{*}(x) \neq 0$.

Proof. Since either $x \notin K$ or $-x \notin K$ if $x \neq 0$, this follows immediately from Theorem 1.

A linear operator $T$ on $B$ into $B$ will be called positive with respect to a cone $K$ if $T K \subseteq K$. In the absence of ambiguity we will simply say $T$ is positive. In our applications $K$ will be a closed cone and in this case, in view of Theorem $1, T$ is positive if and only if $x^{*}(T x) \geqq 0$ for all $x \geqq 0, x^{*} \geqq 0$. Since $T x \geqq 0$ if $x \geqq 0$, we have $x^{*}\left(T^{2} x\right) \geqq 0$ and, in general, $x^{*}\left(T^{n} x\right) \geqq 0$ for all $n$ and all $x \geqq 0, x^{*} \geqq 0$. We define $T$ to be quasi-positive if for each pair $x \geqq 0, x^{*} \geqq 0$, there exists an integer $n\left(x, x^{*}\right) \geqq 1$ such that $x^{*}\left(T^{n} x\right) \geqq 0$ if $n \geqq n\left(x, x^{*}\right)$. We define $T$ to be strictly quasi-positive if for each pair $x>0, x^{*}>0$, there exists an integer $n\left(x, x^{*}\right) \geqq 1$ such that $x^{*}\left(T^{n} x\right)>0$ if $n \geqq$ $n\left(x, x^{*}\right)$. Finally we define $T$ to be strongly quasi-positive if it is not nilpotent ${ }^{3}$ and for each pair $x>0, x^{*}>0, \lim _{n \rightarrow \infty} x^{*}\left(T^{n} x\right) /\left\|T^{n}\right\|>0$.

4. Spectral properties. Throughout this section, $K$ will denote a closed proper cone in $B$ and $K$ will be assumed to be fundamental. $T$ will denote a quasi-compact bounded linear operator with spectral radius 1 . This restriction on the spectral radius is for convenience only and the results given may be interpreted for a general (quasicompact) bounded linear operator $S$ with spectral radius $r_{S}>0$ by considering the operator $T=\left(1 / r_{S}\right) S$ which has spectral radius 1 .

${ }^{3}$ An operator $T$ is nilpotent if $T^{n}=0$ for some $n$. 
THEOREM 2. If $T$ is quasi-positive and quasi-compact with spectral radius 1 , then $1 \in \sigma(T)$ and the index of 1 is not exceeded by the index of any other point $\lambda \in \sigma(T),|\lambda|=1$.

Proof. Assume that $1 \in \rho(T)$. Since $\rho(T)$ is open and $R(\lambda, T)$ is analytic in $\lambda$ for $\lambda \in \rho(T)$, it follows that the function $g(\lambda)=$ $x^{*}(R(1 / \lambda, T) x), x>0, x^{*}>0$, is analytic for $1 / \lambda \in \rho(T)$, in particular for $\lambda$ in some neighborhood of 1 . Moreover, $R(\lambda, T)=\sum_{k=0}^{\infty}(1 / \lambda)^{k+1} \widetilde{T}^{k}$ if $|\lambda|>1$, hence $g(\lambda)=\sum_{k=0}^{\infty} \lambda^{k+1} x^{*}\left(T^{k} x\right)$ if $|\lambda|<1$. A theorem of Pringsheim states that if a power series has nonnegative coefficients and converges in the open unit disk, either 1 is a singularity of the series or the series has radius of convergence greater than $1 .{ }^{4}$ Clearly it is sufficient to assume that all but a finite number of the coefficients are nonnegative. Since $x^{*}\left(T^{n} x\right) \geqq 0$ if $n \geqq n\left(x, x^{*}\right)$, and $g(\lambda)$ is analytic in a neighborhood of 1 , we conclude that the series $\sum_{k=0}^{\infty} \lambda^{k+1} x^{*}\left(T^{k} x\right)$ converges in $|\lambda|<1+\delta$ for some $\delta>0$. By assumption $r_{T}=1$, hence $R(\lambda, T)$ has a singularity somewhere on $|\lambda|=1$, say at $\lambda_{0}$. Since $T$ is quasi-compact, the expansion

$$
R(\lambda, T)=\sum_{k=1}^{n} \frac{\left(\lambda_{0} I-\widetilde{T}\right)^{k-1}}{\left(\lambda-\lambda_{0}\right)^{k}} P\left(\lambda_{0}, T\right)+\sum_{k=0}^{n}\left(\lambda-\lambda_{0}\right)^{k} A_{k}\left(\lambda_{0}, T\right)
$$

is valid for $0<\left|\lambda-\lambda_{0}\right|<\delta^{\prime}$, where $n=n\left(\lambda_{0}\right)$ is the index of $\lambda_{0}$. and $\left(\lambda_{0} I-\widetilde{T}\right)^{n-1} P\left(\lambda_{0}, T\right) \neq 0$. We may choose $x>0$ such that $\left(\lambda_{0} I-\widetilde{T}\right)^{n-1} P\left(\lambda_{0}, T\right) x=y \neq 0$ since $K$ is fundamental and by Theorem 1 we may choose $x^{*}>0$ such that $x^{*}(y) \neq 0$. It follows easily that

$$
g(\lambda)=\left(\lambda / \lambda_{0}\right)^{n}\left(1 / \lambda_{0}-\lambda\right)^{-n} h(\lambda), \quad\left|1 / \lambda-\lambda_{0}\right|<\delta,
$$

where $h(\lambda)$ is analytic and $h\left(1 / \lambda_{0}\right)=x^{*}(y) \neq 0$. Thus $g$ has a pole at $1 / \lambda_{0}$ which contradicts the fact that $g$ has a Taylor's series about the origin with radius of convergence greater than 1 . Our assumption that $1 \in \rho(T)$ leads to a contradiction, hence $1 \in \sigma(T)$.

Now let the index of 1 be $n$. It is easy to see that $\lim _{\lambda \rightarrow 1}(\lambda-1)^{k} R(\lambda, T)=0$ if $k>n$. It follows that for $|\lambda|>1$, $\lim _{\lambda \rightarrow 1}(\lambda-1)^{k} \sum_{m=0}^{\infty}(1 / \lambda)^{m+1} x^{*}\left(T^{m} x\right)=0$ for every pair $x>0, x^{*}>0$ and clearly this implies $\lim _{\lambda \rightarrow 1}(\lambda-1)^{k} \sum_{m=j}^{\infty}(1 / \lambda)^{m+1} x^{*}\left(T^{m} x\right)=0$ if $k>n$ and $j \geqq 0$. If $\lambda_{0} \in \sigma(T),\left|\lambda_{0}\right|=1$ and $\lambda_{0}$ has index $l$, then $\lim _{\lambda \rightarrow \lambda_{0}}\left(\lambda-\lambda_{0}\right)^{l} R(\lambda, T) \neq 0$. We may choose $x>0$ and $x^{*}>0$ such that $\lim _{\lambda \rightarrow \lambda_{0}}\left(\lambda-\lambda_{0}\right)^{l} x^{*}(R(\lambda, T) x) \neq 0$ and it follows that for $|\lambda|>1$, $\lim _{\lambda \rightarrow \lambda_{0}}\left(\lambda-\lambda_{0}\right)^{l} \sum_{m=j}^{\infty}(1 / \lambda)^{m+1} x^{*}\left(T^{m} x\right) \neq 0$. Let $\lambda_{0}=e^{i \varphi}, \lambda=\rho e^{i \varphi}, \rho>1$. If $j \geqq n\left(x, x^{*}\right),\left|\left(\lambda-\lambda_{0}\right)^{l} \sum_{m=j}^{\infty}(1 / \lambda)^{m+1} x^{*}\left(T^{m} x\right)\right| \leqq(\rho-1)^{l} \sum_{m=j}^{\infty}(1 / \rho)^{m+1} x^{*}\left(T^{m} x\right)$. The expression on the right in this last inequality tends to zero as

${ }^{4}$ See Titchmarsh, Theory of Functions, pg. 214. Acknowledgement is due here to S. Karlin for the essence of the proof in Theorem 2 (see [10], Theorem 4). 
$\rho$ tends to 1 if $l>n$, hence $l \leqq n$. This completes the proof.

THEOREM 3. If $T$ is quasi-positive and quasi-compact with spectral radius 1 , there exist elements $u>0$ and $u^{*}>0$ such that $T u=u, T^{*} u^{*}=u^{*} .^{5}$

Proof. By Theorem 2, $1 \in \sigma(T)$. We have

$$
R(\lambda, T)=\sum_{k=1}^{n} \frac{(I-\widetilde{T})^{k-1}}{(\lambda-1)^{k}} P(1, T)+\sum_{k=0}^{\infty}(\lambda-1)^{k} A_{k}(1, T)
$$

where $P(1, T)$ is a projection onto the finite-dimensional space $\left\{x \mid(I-\widetilde{T})^{n} x=0\right\}$ and $(I-\widetilde{T})^{n-1} P(1, T) \neq 0$. Let $\Gamma=(I-\widetilde{T})^{n-1} P(1, T)$. It is easy to see that $R(\lambda, T) B \cong B$ for $\lambda$ real. Since $\Gamma=$ $\lim _{\lambda \rightarrow 1}(\lambda-1)^{n} R(\lambda, T)$, it follows that $\Gamma B \subseteq B$. Also $\widetilde{T} \Gamma=\Gamma \widetilde{T}=\Gamma$. Let $x \geqq 0, x^{*} \geqq 0$ be arbitrary and let $N=n\left(x, x^{*}\right)$. If $\lambda>1$, we have $x^{*}\left(T^{N} R(\lambda, T) x\right)=\sum_{m=0}^{\infty}(1 / \lambda)^{m+1} x^{*}\left(T^{N+m}\right) x \geqq 0$. It follows that for $\lambda>1, x^{*}\left(T^{N} \Gamma x\right)=\lim _{\lambda \rightarrow 1}(\lambda-1)^{n} \sum_{m=0}^{\infty}(1 / \lambda)^{m+1} x^{*}\left(T^{N+m} x\right) \geqq 0$. Since $T^{N} \Gamma=\Gamma, \Gamma$ is a positive operator. We choose $v>0$ such that $\Gamma v=$ $u \neq 0$. Then $u>0$ and $T u=T \Gamma v=\Gamma v=u$. We choose $v^{*}>0$ such that $v^{*}(u)>0$. Letting $u^{*}=\Gamma^{*} v^{*}$, we see that for $x \geqq 0, u^{*}(x)=$ $\left(\Gamma^{*} v^{*}\right)(x)=v^{*}(\Gamma x) \geqq 0$ since $v^{*}>0$ and $\Gamma$ is a positive operator. Hence $u^{*} \geqq 0$, and since $u^{*}(v)=\left(\Gamma^{*} v^{*}\right)(v)=v^{*}(\Gamma v)=v^{*}(u)>0, u^{*}>0$. Finally, we have $\Gamma T=\Gamma$ which implies $T^{*} \Gamma^{*}=\Gamma^{*}$, hence $T^{*} u^{*}=$ $T^{*}\left(\Gamma^{*} v^{*}\right)=\Gamma^{*} v^{*}=u^{*}$ which completes the proof.

For strictly quasi-positive operators we obtain stronger results in the next two theorems.

THEOREM 4. If $T$ is strictly quasi-positive and quasi-compact with spectral radius 1 , then $1 \in \sigma(T), 1$ has index one and $\widetilde{T}$ has a representation of the form $\widetilde{T}=\sum_{j=1}^{m} \lambda_{j} P_{j}+S$ where $\lambda_{1}=1,\left|\lambda_{j}\right|=1$, $P_{\jmath}^{2}=P_{j}, \quad S P_{j}=P_{j} S=0, \quad j=1,2, \cdots, m, \quad P_{i} P_{j}=0 \quad$ if $i \neq j$, and $r_{s}<1$.

Proof. By Theorem 2, $1 \in \sigma(T)$. By Theorem 3, there exists $u^{*}>0$ such that $T^{*} u^{*}=u^{*}$ and for $x>0, u^{*}(x)=u^{*}\left(T^{n} x\right)>0$ if $n \geqq n\left(x, u^{*}\right)$, hence $u^{*}$ is strictly positive. Let the index of 1 be $n$. Then $\Gamma=\lim _{\lambda \rightarrow 1}(\lambda-1)^{n} R(\lambda, T) \neq 0$. For $\lambda>1$ and arbitrary $x$ we have

$$
\begin{aligned}
u^{*}(\Gamma x) & =\lim _{\lambda \rightarrow 1}(\lambda-1)^{n} \sum_{k=0}^{\infty}(1 / \lambda)^{k+1} u^{*}\left(T^{k} x\right)=\lim _{\lambda \rightarrow 1} u^{*}(x)(\lambda-1)^{n} \sum_{k=0}^{\infty}(1 / \lambda)^{k+1} \\
& =u^{*}(x) \lim _{\lambda \rightarrow 1}(\lambda-1)^{n-1}=0
\end{aligned}
$$

${ }^{5} T^{*}$ is the adjoint of $T$, defined on $B^{*}$ by $\left(T^{*} x^{*}\right)(x)=x^{*}(T x)$. 
unless $n=1$. In proving Theorem 3 we showed that $\Gamma$ is a positive operator, hence there exists $x>0$ such that $\Gamma x>0$ and therfore $u^{*}(\Gamma x)>0$. It follows that $n=1$. By Theorem 2, every $\lambda_{0} \in \sigma(T)$, $\left|\lambda_{0}\right|=1$, has index 1 and hence $P\left(\lambda_{0}, T\right)=\lim _{\lambda \rightarrow \lambda_{0}}\left(\lambda-\lambda_{0}\right) R(\lambda, T)$ exists and is a projection onto the finite dimensional space $\left\{x \mid\left(\lambda_{0} I-\widetilde{T}\right) x=0\right\}$. Let $\lambda_{1}=1, \lambda_{2} \cdots, \lambda_{m}$ be an enumeration of the points in $\sigma(T)$ with absolute value 1 and let $P_{j}=P\left(\lambda_{j}, T\right)$. Since $\widetilde{T}$ commutes with $R(\lambda, T)$ and $P_{j}=\lim _{\lambda \rightarrow \lambda_{j}}\left(\lambda-\lambda_{j}\right) R(\lambda, T)$, it follows that $\widetilde{T}$ commutes with $P_{j}$. For $i \neq j$ we have $\lambda_{i} P_{i} P_{j}=\widetilde{T} P_{i} P_{j}=P_{i} \widetilde{T} P_{j}=\lambda_{j} P_{i} P_{j}$, hence $P_{i} P_{j}=0$. Define the bounded linear operator $S$ by the equation $\widetilde{T}=\sum_{j=1}^{m} \lambda_{j} P_{j}+S$. Since $\widetilde{T} P_{j}=P_{j} \widetilde{T}=\lambda_{j} P_{j}, \quad P_{j}^{2}=P_{j}$ and $P_{i} P_{j}=0$ if $i \neq j$, it follows that $P_{j} S=S P_{j}=0$. This implies $\widetilde{T}^{n}=\sum_{j=1}^{m} \lambda_{j}^{n} P_{j}+S^{n}$. Suppose $r_{S} \geqq$ 1. $T$ is quasi-compact, hence $\widetilde{T}^{n}=U+V$ for some $n$ where $U$ is compact and $r_{V}<1$. The operator $U^{\prime}$ defined by $U^{\prime} x=U x-\sum_{j=1}^{m} \lambda_{j}^{n} P_{j} x$ is compact ${ }^{6}$ and $S^{n}=U^{\prime}+V$. Therefore $S$ is quasi-compact. Let $\lambda \in \sigma(S),|\lambda|=r_{S} \geqq 1$. Then $S x=\lambda x$ for some $x \in \widetilde{B}, x \neq 0$. Since $P_{j} S=S P_{j}=0$, it follows that $\widetilde{T} x=\lambda x$ and therefore for some $j, \lambda=\lambda_{j}$ and $P_{j} x=x$. This implies $S x=S P_{j} x=0$, a contradiction. Therefore $r_{S}<1$ and the proof is complete.

Before stating our next result, we state the following lemma which is easily proved.

LEMMA 1. If $E$ is a finite dimensional real Banach space, $K$ is a cone in $E$ and $K$ is fundamental, then $K$ contains an open set.

THEOREM 5. If $T$ is strictly quasi-positive and quasi-compact with spectral radius 1 , the eigenspace for $T$ corresponding to the eigenvalue 1 is one-dimensional.

Proof. By Theorem 4 we have $\widetilde{T}=\sum_{j=1}^{m} \lambda_{j} P_{j}+S$ where $P_{j}$ is a projection onto the eigenspace corresponding to $\lambda_{j}, \lambda_{1}=1,\left|\lambda_{j}\right|=1$, $P_{j} S=S P_{j}=0, j=1,2, \cdots, m$ and $P_{i} P_{j}=0$ if $i \neq j$. By a theorem of Kronecker, there exists a sequence $n_{1}, n_{2} \cdots$ of positive integers such that $\lim _{k \rightarrow \infty} \lambda_{j}^{n} k=1, j=1,2, \cdots, m .^{7}$ Since $r_{s}<1$, it follows that $\lim _{n \rightarrow \infty}\left\|S^{n}\right\|=0$. This implies $\lim _{k \rightarrow \infty} \widetilde{T}^{n_{k}}=\sum_{j=1}^{m} P_{j}$. Let $P=$ $\sum_{j=1}^{m} P_{j}$. For $x \in B$ we have $P x=\lim _{k-\infty} T^{n_{k}} x$, hence $P B \subseteq B$. For $x \geqq 0$ and $x^{*} \geqq 0, x^{*}(P x)=\lim _{k \rightarrow \infty} x^{*}\left(T^{n_{k}} x\right) \geqq 0$, hence $P$ is a positive operator. Consider the finite dimensional real Banach space $P B$ with closed proper cone $P K$. Since $K$ is fundamental in $B$, it is clear that $P K$ is fundamental in $P B$. Therefore, by Lemma $1, P K$ contains an open set (open relative to $P B$ ). Since $T$ is strictly quasi-positive, every

${ }_{6}^{6}$ The compact operators from an ideal in the algebra of bounded linear operators and any bounded operator with a finite dimensional range is compact.

${ }^{7}$ See, for example, Hardy \& Wright, The Theory of Numbers, Oxford Univ. Press. 
non-trivial fixed vector of $T$ in $K$ is strictly positive. By Theorem 3, there exists $u>0$ such that $T u=u$. Let $T x=x, x \neq 0$. We wish to show $u$ and $x$ are linearly dependent and for this purpose we may assume $x \notin K$ (otherwise replace $x$ by $-x$ ). It is clear that $u \in P K$ and $x \in P B$. Let $t_{0}=\sup \{t \mid u+t x \in P K\}$. Since $u$ is in the interior of $P K$ and $x \notin P K$, it is easy to see that $0<t_{0}<\infty$ and that $u+t_{0} x$ is on the boundary of $P K$. Hence, by Theorem 1 , there exists $x^{*} \in(P K)^{+}$ such that $x^{*}\left(u+t_{0} x\right)=0$. We extend $x^{*}$ to $y^{*} \in B^{*}$ by defining $y^{*}(y)=$ $x^{*}(P y)$. Since $P K \subseteq K$, it follows that $y^{*} \in K^{+}$. We have $P\left(u+t_{0} x\right)=$ $u+t_{0} x$, hence $y^{*}\left(u+t_{0} x\right)=x^{*}\left(u_{0}+t_{0} x\right)=0$. Now $u+t_{0} x$ is a fixed vector of $T$ which is not strictly positive, hence $u+t_{0} x=0$, which completes the proof.

Our next result is a characterization of strongly quasi-positive operators.

THEOREM 6. If $T$ is quasi-compact with spectral radius 1 , then $T$ is strongly quasi-positive if and only if the following conditions are satisfied:

(1) $1 \in \sigma(T)$ and 1 is the only point in $\sigma(T)$ with absolute value one,

(2) the eigenspace for $T$ corresponding to the eigenvalue 1 is one-demensional and is spanned by a strictly positive element $u$,

(3) there exists a strictly positive element $u^{*}$ such that $T^{*} u^{*}=$ $u^{*}$.

Proof. In Theorems 3, 4, 5 we have seen that if $T$ is strictly quasi-positive (in particular, if it is strongly quasi-positive), then $1 \in \sigma(T)$ and (2) and (3) hold. There remains to show 1 is the only point in $\sigma(T)$ with absolute value one. We define the operator $P=$ $\sum_{j=1}^{m} P_{j}$ as in Theorem 5 and recall that $P B$ is a finite dimensional real Banach space with closed proper cone $P K$ containing interior elements. Let $\lambda=e^{i \theta}$ be a point in $\sigma(T)$ and let $\widetilde{T}(x+i y)=e^{i \theta}(x+i y)$ for some $x, y$ in $B$, not both zero. It is easy to see that $P x=x$ and $P y=y$, hence $x \in P B$ and $y \in P B$. At least one of the four elements $x+y, x-y, y-x,-x-y$ must be not in $P K$ since otherwise $x+y=0, x-y=0$, hence $x=y=0$. Therefore $a x+b y \notin P K$ for some choice of $a= \pm 1$ and $b= \pm 1$. Now choose $t>0$ such that $u+t(a x+b y)=v$ is on the boundary of $P K$. By Theorem 1 , there exists $x^{*} \in(P K)^{+}, x^{*} \neq 0$, such that $x^{*}(v)=0$. We extend $x^{*}$ to $y^{*} \in K^{+}: y^{*}(y)=x^{*}(P y)$. Now choose a sequence of positive integers $n_{1}, n_{2}, \cdots$ such that $\lim _{k \rightarrow \infty} e^{i n_{k} \theta}=1$. It follows that $\lim _{k \rightarrow \infty} T^{n_{k}} v=v$. Since $r_{T}=1$, we have $\left\|T^{n}\right\| \geqq 1$ for all $n$ and hence if $v>0$,

$$
\lim \inf _{n \rightarrow \infty} y^{*}\left(T^{n} v\right) \geqq \lim \inf _{n \rightarrow \infty} y^{*}\left(T^{n} v\right) /\left\|T^{n}\right\|>0 .
$$


This is impossible since $\lim _{k \rightarrow \infty} y^{*}\left(T^{n_{k}} v\right)=y^{*}(v)=0$. Therefore $v=0$, i.e., $a x+b y=-(1 / t) u$. Since $\widetilde{T}(x+i y)=e^{i \theta}(x+i y)$, it follows that $u^{*}(x)+i u^{*}(y)=e^{i \theta}\left(u^{*}(x)+i u^{*}(y)\right)$. This implies either $e^{i \theta}=1$ or $u^{*}(x)=u^{*}(y)=0$. The second alternative is incompatible with $a x+b y=-(1 / t) u$ since $u^{*}(u)>0$. Therefore $e^{i \theta}=1$ and the necessity of (1), (2), (3) is proved.

Now let $T$ satisfy conditions (1), (2), (3). We assume without loss of generality that $u^{*}$ is normalized so that $u^{*}(u)=1$. Define the bounded linear operator $S$ by $T x=u^{*}(x) u+S x$. As in Theorem 4, it can be shown that $r_{S}<1$. We have $S u=T u-u^{*}(u) u=u-u=0$ and it follows that $T^{n} x=u^{*}(x) u+S^{n} x$. Since $r_{S}<1,\left\|S^{n}\right\| \leqq M$ for all $n$ and hence $\left\|T^{n}\right\| \leqq\left\|u^{*}\right\|\|u\|+\left\|S^{n}\right\| \leqq M^{\prime}$ for all $n$. Moreover, $S^{n} x \rightarrow 0$ as $n \rightarrow \infty$ for all $x$. Hence if $x>0$ and $x^{*}>0$,

$$
\begin{aligned}
\lim \inf _{n \rightarrow \infty} x^{*}\left(T^{n} x\right) /\left\|T^{n}\right\| & \geqq \lim \inf _{n \rightarrow \infty}\left(u^{*}(x) x^{*}(u)+x^{*}\left(S^{n} x\right)\right) / M^{\prime} \\
& \geqq u^{*}(x) x^{*}(u) / M^{\prime}>0 .
\end{aligned}
$$

Therefore $T$ is strongly quasi-positive and the theorem is proved.

THEOREM 7. Assume that $B$ is a lattice ${ }^{8}$ with respect to the ordering given by $K$. Then Theorem 6 is true if "strongly quasipositive" is replaced by "strictly quasi-positive."

Proof. Conditions (1), (2) and (3) in Theorem 6 imply $T$ is strongly quasi-positive, hence, a fortiori, $T$ is strictly quasi-positive. Now suppose $T$ is strictly quasi-positive. Then $1 \in \sigma(T)$ and (2), (3) hold. It is easy to see from the representation of Theorem $4, \widetilde{T}=\sum_{j=1}^{m} \lambda_{j} P_{j}+$ $S$, that $\left\|T^{n}\right\|$ is bounded independently of $n$. Hence, by a theorem of Krein-Rutman ([11], Theorem 8.1 and corollary), every $\lambda \in \sigma(T)$, $|\lambda|=1$, is a root of unity. It is easily verified that every power of $T$ is quasi-compact and strictly quasi-positive, hence the eigenspace for $T^{n}$ corresponding to the eigenvalue 1 is one-dimensional for all $n$. If $\widetilde{T} x=\lambda x,|\lambda|=1, \lambda^{n}=1$, then $\widetilde{T}^{n} x=\lambda^{n} x=x$ and it follows that $\lambda=1$ which completes the proof.

An immediate consequence is the following corollary.

Corollary. If $B$ is a lattice, every strictly quasi-positive and quasi-compact operator is strongly quasi-positive.

The conclusion of this corollary is not true in general as we will illustrate by an example. Let $B$ be three-dimensional (real) Euclidean bound.

${ }^{8}$ I.e., each pair of elements in $B$ has a greatest lower bound and a least upper 
space, $B=\left\{\left(x_{1}, x_{2}, x_{3}\right)\right\}$, and let $K=\left\{\left(x_{1}, x_{2}, x_{3}\right) \mid x_{1}^{2}+x_{2}^{2} \leqq x_{3}^{2}, x_{3} \geqq 0\right\}$. If we interpret "to the right" to mean any direction in which the $x_{3}$ coordinate is increasing, each non-trivial element $x^{*} \in K^{+}$is represented by a plane through the origin whose unit normal at the origin directed to the right lies in $K$. Let $T$ be a rotation about the $x_{3}$ axis through $\theta$ radians where $\theta$ and $2 \pi$ are incommensurable. It is clear that $\left\|T^{n}\right\|=1$ for all $n$ and that $T K \subseteq K$. To show that $T$ is strictly quasi-positive it suffices to consider $x^{*} \in K^{+}$which is represented by a plane tangent to $K$. If $p$ is in the interior of $K, T^{n} p$ is in the interior for all $n$, hence $x^{*}\left(T^{n} p\right)>0$. Now let $p$ be on the boundary of $K$. There exists exactly one point $q$ which has the same $x_{3}$ coordinate as $p$ and such that $x^{*}(q)=0$. Since $\theta$ and $2 \pi$ are incommensurable, there is at most one value of $n$ such that $T^{n} p=q$. Therefore, $x^{*}\left(T^{m} p\right)>0$ for all $m$ sufficiently large and, hence, $T$ is strictly quasi-positive. If $p$ is on the boundary of $K$, so is $T^{n} p$ for all $n$. We can pick a sequence $n_{1}, n_{2}, \cdots$ such that $T^{n^{n}} p$ converges to a point $q$ on the boundary of $K$ and there exists $x^{*} \in K^{+}$such that $x^{*}(q)=0, x^{*} \neq 0$. This shows $T$ is not strongly quasi-positive.

\section{BIBLIOGRAPHY}

1. T. Ando, Positive linear operators in semi-ordered linear spaces, J. Fac. Science, Hokkaido University, Ser. I, XIII, (1957), 214-228.

2. G. Birkhoff, Extensions of Jentzsch's thoorem, Trans. Amer. Math. Soc., 85 (1957), 219-228.

3. F. F. Bonsall, Endomorphisms of partially ordered vector spaces, J. London Math. Soc., 30 (1955), 133-144.

4. —- Endomorphism of a partially ordered vector space without order unit, J. London Math. Soc., 30 (1955), 144-153.

5 . L Linear operators in complete positive cones, Proc. London Math. Soc., 3, VIII (29), (1958), 53-75.

6. G. Frobenius, Über Matrizen aus positiven Elementen, Sitz. Ber. Preuss. Academie der Wiss. Berlin (1908), 471-476, (1909), 514-518.

7. _— Über Matrizen aus nicht negativen Elementen, Sitz. Ber. Preuss. Akademie der Wiss. Berlin (1912), 456-477.

8. E. Hopf, Über lineare Integralgleichungen mit positiven Kern, Sitz. Ber. Preuss. Akademie der Wiss. Berlin, XVIII (1928), 233-245.

9. R. Jentzsch, Über Integralgleichungen mit positiven Keri, Crelles J., 141 (1912), 235-244.

10. S. Karlin, Positive Operators, J. Math., 8 (1959), 907-938.

11. M. Krein and M. Rutman, Linear operators leaving invariant a cone in a Banach. space, Uspehi Matem. Navk (1948), 3-95, Amer. Math. Soc. Translation No. 26.

12. O. Perron, Zur Theorie der Matrizen, Math. Ann., 64 (1907), 248-263.

13. H. Schaefer, Positive Transformationen in lokal konvexen halbgeordneten Velctorräumen, Math. Ann., 129 (1955), 323-329.

14. - Some spectral properties of positive linear operators, Pacific J. Math., 10 (1960), 1009-1019. 



\section{PACIFIC JOURNAL OF MATHEMATICS}

\section{EDITORS}

\author{
Robert Osserman \\ Stanford University \\ Stanford, California
M. G. Arsove
University of Washington
Seattle 5 , Washington

\author{
J. DugundJI \\ University of Southern California \\ Los Angeles 7, California
}

Lowell J. Paige

University of California

Los Angeles 24, California

\section{ASSOCIATE EDITORS}
E. F. BECKENBACH
B. H. NeumanN
F. WOLF
K. YOSIDA

\section{SUPPORTING INSTITUTIONS}

\author{
UNIVERSITY OF BRITISH COLUMBIA \\ CALIFORNIA INSTITUTE OF TECHNOLOGY \\ UNIVERSITY OF CALIFORNIA \\ MONTANA STATE UNIVERSITY \\ UNIVERSITY OF NEVADA \\ NEW MEXICO STATE UNIVERSITY \\ OREGON STATE UNIVERSITY \\ UNIVERSITY OF OREGON \\ OSAKA UNIVERSITY \\ UNIVERSITY OF SOUTHERN CALIFORNIA
}

\author{
STANFORD UNIVERSITY \\ UNIVERSITY OF TOKYO \\ UNIVERSITY OF UTAH \\ WASHINGTON STATE UNIVERSITY \\ UNIVERSITY OF WASHINGTON \\ * * * * \\ AMERICAN MATHEMATICAL SOCIETY \\ CALIFORNIA RESEARCH CORPORATION \\ SPACE TECHNOLOGY LABORATORIES \\ NAVAL ORDNANCE TEST STATION
}

Mathematical papers intended for publication in the Pacific Journal of Mathematics should by typewritten (double spaced), and on submission, must be accompanied by a separate author's résumé. Manuscripts may be sent to any one of the four editors. All other communications to the editors should be addressed to the managing editor, L. J. Paige at the University of California, Los Angeles 24, California.

50 reprints per author of each article are furnished free of charge; additional copies may be obtained at cost in multiples of 50 .

The Pacific Journal of Mathematics is published quarterly, in March, June, September, and December. Effective with Volume 13 the price per volume (4 numbers) is $\$ 18.00$; single issues, $\$ 5.00$. Special price for current issues to individual faculty members of supporting institutions and to individual members of the American Mathematical Society: $\$ 8.00$ per volume; single issues $\$ 2.50$. Back numbers are available.

Subscriptions, orders for back numbers, and changes of address should be sent to Pacific Journal of Mathematics, 103 Highland Boulevard, Berkeley 8, California.

Printed at Kokusai Bunken Insatsusha (International Academic Printing Co., Ltd.), No. 6, 2-chome, Fujimi-cho, Chiyoda-ku, Tokyo, Japan.

PUBLISHED BY PACIFIC JOURNAL OF MATHEMATICS, A NON-PROFIT CORPORATION

The Supporting Institutions listed above contribute to the cost of publication of this Journal, but they are not owners or publishers and have no responsibility for its content or policies. 


\section{Pacific Journal of Mathematics}

\section{Vol. 14, No. $3 \quad$ July, 1964}

Erik Balslev and Theodore William Gamelin, The essential spectrum of a class of ordinary differential operators . . . . . . . . . . . . . . . . . . . .

James Henry Bramble and Lawrence Edward Payne, Bounds for derivatives in

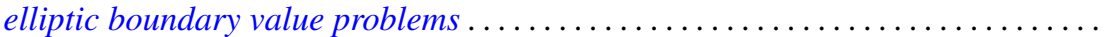

Hugh D. Brunk, Integral inequalities for functions with nondecreasing

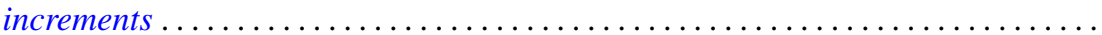

William Edward Christilles, A result concerning integral binary quadratic

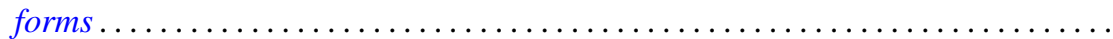

Peter Crawley and Bjarni Jónsson, Refinements for infinite direct decompositions of

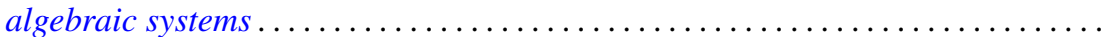

Don Deckard and Carl Mark Pearcy, On continuous matrix-valued functions on a Stonian space.

Raymond Frank Dickman, Leonard Rubin and P. M. Swingle, Another

characterization of the $n$-sphere and related results $\ldots \ldots \ldots \ldots \ldots \ldots$

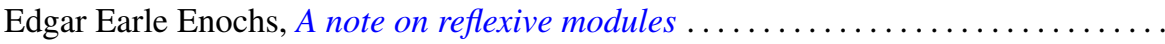

Vladimir Filippenko, On the reflection of harmonic functions and of solutions of the

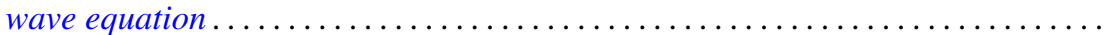

Derek Joseph Haggard Fuller, Mappings of bounded characteristic into arbitrary

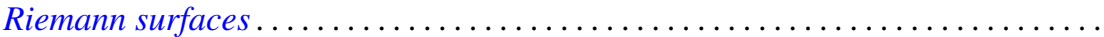
895

Curtis M. Fulton, Clifford vectors . . . . . . . . . . . . . . . . . . . . . . . . . . . . . . 917

Irving Leonard Glicksberg, Maximal algebras and a theorem of Radó . .

919

Kyong Taik Hahn, Minimum problems of Plateau type in the Bergman metric

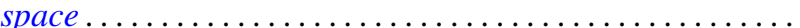

A. Hayes, A representation theory for a class of partially ordered rings...

J. M. C. Joshi, On a generalized Stieltjes trasform

J. M. C. Joshi, Inversion and representation theorems for a generalized Laplace transform ...

Eugene Kay McLachlan, Extremal elements of the convex cone $B_{n}$ of functions ...

Robert Alan Melter, Contributions to Boolean geometry of p-rings ...

James Ronald Retherford, Basic sequences and the Paley-Wiener criterion . . . . . . . 1019

Dallas W. Sasser, Quasi-positive operators. .

Oved Shisha, On the structure of infrapolynomials with prescribed coefficients ..

Oved Shisha and Gerald Thomas Cargo, On comparable means

Maurice Sion, A characterization of weak ${ }^{*}$ convergence ........

Morton Lincoln Slater and Robert James Thompson, A permanent inequality for

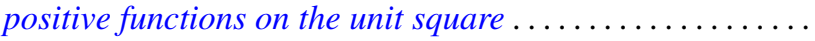

David A. Smith, On fixed points of automorphisms of classical Lie algebras ...

Sherman K. Stein, Homogeneous quasigroups ................

J. L. Walsh and Oved Shisha, On the location of the zeros of some infrapolynomials with prescribed coefficients .

Ronson Joseph Warne, Homomorphisms of $d$-simple inverse semigroups with identity . 